\title{
Corpus
}

\section{Polysignifiance du toponyme, historicité du sens et interprétation en corpus. Le cas de Outreau}

Michelle Lecolle

\section{(2) OpenEdition}

1 Journals

Édition électronique

URL : http://journals.openedition.org/corpus/1122

DOI : $10.4000 /$ corpus. 1122

ISSN : 1765-3126

Éditeur

Bases; corpus et langage - UMR 6039

Édition imprimée

Date de publication : 1 décembre 2007

Pagination : 101-125

ISSN : 1638-9808

\section{Référence électronique}

Michelle Lecolle, « Polysignifiance du toponyme, historicité du sens et interprétation en corpus. Le cas de Outreau », Corpus [En ligne], 6 | 2007, mis en ligne le 02 juillet 2008, consulté le 08 septembre 2020. URL : http://journals.openedition.org/corpus/1122 ; DOI : https://doi.org/10.4000/corpus.1122 


\title{
Polysignifiance du toponyme, historicité du sens et interprétation en corpus. Le cas de Outreau*
}

\author{
Michelle LECOLLE \\ Université Paul Verlaine - Metz, CELTED
}

Notre contribution présente une étude de cas: nous nous centrons sur l'analyse du changement de sens du nom propre Outreau, et sur ce qui éclaire son interprétation en contexte.

Ce nom renvoie, on le sait, à un événement contemporain dramatique qui a pour cadre la ville d'Outreau (Pas-de-Calais), lieu de la « découverte » (par la suite réfutée) d'un réseau pédophile. Au nom propre ( $\mathrm{Npr}$ désormais) Outreau s'attache donc, par des récits et des nominations, l'histoire d'une croyance collective, et de la réfutation collective ultérieure de cette croyance (sanctionnée par un procès en appel en décembre 2005). De cette histoire, rapportée dans les médias, le Npr lui-même porte la trace : un signifiant inchangé voit son sens et sa référence évoluer totalement : ainsi, Outreau est passé d'un sens locatif (/ville/) à celui, stabilisé (en 2005 et par la suite), de /fiasco judiciaire/ - /l'erreur judiciaire par excellence/. En traversant des étapes successives et en prenant différentes valeurs sémantico-référentielles, Outreau est donc devenu en quelque sorte un parangon.

Notre travail s'attache à cerner les modalités du changement de sens du Npr Outreau. Ici, l'essentiel de notre propos sera de caractériser nos critères d'analyse et nos méthodes de repérage des différents sens. Comme nous y invite le cadrage de ce numéro de Corpus, l'approche est à la fois pratique et théorique. Pratique, car il s'agit, dans une étude de cas, de cerner concrètement les éléments pertinents qui

* Merci à Marie-Paule Jacques et à Josette Rebeyrolle pour leur relecture d'une première version de cet article et pour leurs conseils amicaux. Merci également aux deux relecteurs anonymes pour leurs remarques constructives et éclairantes.

Corpus n ${ }^{\circ} 6$ « Interprétation, contextes, codage» (2007), 101-125 
permettent de décrire l'évolution du sens et de la référence d'un toponyme, et d'un toponyme spécifique, largement tributaire de circonstances historiques spécifiques. Théorique car, au-delà du cas du toponyme, et d'un toponyme particulier, nous chercherons à tirer des enseignements plus généraux sur le rôle du contexte et le regard de l'analyste "outillé ${ }^{1}$ » pour l'interprétation d'un nom dont le sens évolue, mais qui est aussi, dès le départ, polysignifiant (ce qui renvoie plus généralement à la question de l'interprétation de la polysémie et à la désambiguïsation si l'on veut).

Afin d'élargir à bon escient notre analyse, nous commencerons, dans une première partie, par présenter ce qui, dans notre étude, tient au cas du Npr. La spécificité théorique du Npr comme nom d'individu, et particulièrement celle du nom de lieu habité, a en effet des incidences sur l'analyse - sur les méthodes tout autant que sur les résultats obtenus.

La partie suivante présentera le choix du corpus. Depuis les critères de sélection de ce corpus jusqu'à sa partition (chronologique), en passant par la méthode de recueil, ce choix est déjà un geste interprétatif. Il est guidé, notamment, par le souci d'une approche historique du sens du Npr Outreau, éclairé par la connaissance contemporaine de ce sens (en 2006). Ce corpus est considéré comme le contexte - non auto-suffisant - permettant de mettre en présence tout à la fois les éléments qui font évoluer le sens de Outreau, et les différents sens euxmêmes du Npr.

La suite de l'article présentera les angles d'analyse choisis pour cerner, sous différents éclairages, l'interprétation de Outreau dans les différentes périodes retenues. Ces angles d'analyse conduisent à une interprétation directement ou, le plus souvent, indirectement, en appui d'autres faits contextuels. Dans le premier cas, ils ont pour nous une fonction de "marqueur » (comme la présence de certains mots ou de certaines structures), et dans le second d'indice, ou de guide pour l'interprétation. Nous réservons les dernières parties pour discuter ces points, en nous centrant sur quelques exemples significatifs.

1. Nous avons employé le concordancier Antconc. 
Polysignifiance du toponyme

Le contexte intervient ici dans toutes les phases de l'analyse. Il est conçu comme le lieu de la construction d'affinités mutuelles de sens, qui maintient parfois des zones de chevauchement et d'indétermination et non, du moins a priori, comme filtrant ou désambiguïsant ${ }^{2}$. C'est ainsi, nous semble-til, que peut être décrite l'évolution du sens d'un Npr, qui passe par des zones intermédiaires et continues de chevauchement des sens.

\section{Nom propre, toponyme}

\subsection{Toponyme et polysignifiance}

A rebours d'une conception du Npr qui s'attache à une univocité définitoire, d'ailleurs déjà largement remise en question $^{3}$, plusieurs études récentes portant sur le toponyme ${ }^{4}$, ou plus spécifiquement sur le nom de lieu habité, mettent en évidence sa polysignifiance intrinsèque et sa polyvalence discursive. Cislaru (2005) parle à son propos d'« omnisignifiance ». En adoptant, pour notre part, le terme de "polysignifiance ", nous souscrivons, de par nos propres observations, aux attendus de ses analyses. Ainsi, le Npr de lieu habité, tout en portant, comme tout Npr, une instruction d'unicité, présente différentes valeurs sémantico-référentielles : il renvoie au lieu, mais aussi aux habitants et à l'institution qui le gouverne (pays ou commune). Ces différentes valeurs, normalement discriminées en contexte, ne sont en fait pas nécessairement exclusives dans une occurrence donnée, où elles se conjuguent parfois ${ }^{5}$. En outre, le toponyme peut, en contexte, recevoir par métonymie une valeur événementielle susceptible de se stabiliser (c'est le cas connu de Tchernobyl), voire

2. Nous nous appuyons sur les propositions de Fuchs (notamment 1991).

3. Et notamment par Paul Siblot. Dans Siblot (1997) « D'un nom à l'autre », Nouvelle revue d'onomastique 29/30, p. 3-18, cité par Cislaru (2005), on relève (p. 15) : "Le Npr s'avère apte à véhiculer des représentations différenciées, voire contradictoires, de l'individu désigné ». Cf. aussi, plus anciennement, Gary-Prieur (1994), et plus généralement les approches du « Npr modifié » présentées dans Leroy (2004).

4. Cislaru (2005), Lecolle (2004, 2006, à paraître).

5. Cf. sur ce point Lecolle (2004 et 2006). 
d'autres valeurs encore, acquises dans son histoire - et c'est, on le sait, ce qui se passe pour Outreau (cf. infra).

Résumons : à un être unique, auquel est attribué, de manière stable, un $\mathrm{Npr}$, se rattachent plusieurs facettes. L'unicité de cet être - le référent du $\mathrm{Npr}$ - lui vaut précisément le cumul de ces facettes, que ce soit dans son sémantisme originel ou de par son histoire. Le nom de lieu habité possède ainsi une grande richesse sémantique.

Du fait de cette polysignifiance, il est tout particulièrement perméable au contexte: son sens n'est nullement figé. Il possède une grande malléabilité et il évolue ; c'est pourquoi il gagne à être étudié en corpus.

\subsection{Signification du nom propre, sens et changement de sens du nom propre}

Pour parler d'évolution du sens du Npr, nous nous fonderons sur l'opposition signification/sens. La signification est de l'ordre du type, et le sens de l'ordre de l'occurrence en contexte.

Le Npr, même inconnu des locuteurs (que ce soit du point de vue de son signifiant ou de celui de son référent), possède une signification catégorielle (/lieu/, /personne/ notamment, /événement/), qui a une contrepartie « en langue » liée à sa substituabilité (on peut remplacer Outreau par la ville), et à son insertion syntagmatique. Une signification minimale, de l'ordre du classème, permet par exemple de prévoir qu'un syntagme prépositionnel (Sprép désormais) comme à Outreau n’est pas interprété préférentiellement comme un datif (cf. à Marie), mais plutôt comme un locatif, qu'on peut trouver et interpréter sans inférence spécifique des expressions comme habiter à Outreau, habiter Outreau.

Mais dès lors qu'il est inséré dans des discours - et, pour Outreau, dans une histoire, des récits -, le Npr relève d'une autre catégorie de "signifiance ». En s'enrichissant des connaissances rapportées à son propos, son sens évolue jusqu'à acquérir une nouvelle signification, susceptible à terme d'être partagée de manière interdiscursive, mais en passant par des zones de turbulence et d'instabilité. C'est ainsi que à Outreau peut renvoyer alors à une toute autre interprétation que /ville/ 
Polysignifiance du toponyme

(cf. [1]), voire demeurer indécidable ; qu'on peut trouver les Sprép après Outreau, malgré Outreau, qui relèvent d'une interprétation événementielle [2].

[1] Les institutions craignent tant d'être accusées de " couvrir » des faits odieux, ou du moins de ne pas enquêter avec ardeur, que personne n'ose prendre de risques. On ouvre souvent le parapluie largement et on laisse à la cour d'assises le soin de faire le tri entre coupables et innocents. Quitte, comme à Outreau, à maintenir des gens en détention et à ruiner des réputations. (Le Figaro, 05/2004)

[2] Les «Outreau " quotidiens de la justice (article du Monde 24/06/06 à propos du livre Le Dossier noir de l'instruction). Commentaire sur ce livre, Eyrolles.com : « Après Outreau, les langues se délient. Ce témoignage de 30 avocats fait froid dans le dos sur les méthodes de la justice: garde-à-vue punitives, experts véreux, erreur de jugement défendue bec et ongles par solidarité entre confrères... Bref, le scandale judiciaire, un mal quotidien. »

A terme donc, c'est par le biais des emplois du Npr, de ses occurrences, que le type est susceptible d'évoluer; le Npr dénote alors un autre individu - on connaît le cas de la métonymie qui fait passer un Npr de la signification /lieu/ à la signification /produit fait dans ce lieu/ (camembert). Le type premier n'en est pas pour autant toujours annulé, et on se trouve alors dans des cas de polysémie et de polyvalence référentielle.

\section{Histoire de Outreau et choix du corpus}

\subsection{Présentation du corpus}

Parmi les Npr jouissant de notoriété et les histoires qui s'y rattachent, le cas de Outreau est un cas d'espèce, et ceci à plusieurs titres :

- le fait "Outreau» est un événement public contemporain, dont on peut trouver la description dans la presse actuelle (depuis 2001-2002 jusqu'à nos jours). A ce titre, la connaissance supposée extra-linguistique du référent du Npr, se trouve, de fait, ramenée à l'observation de faits linguistiques dans un cadre intertextuel ${ }^{6}$.

6. Nous rejoignons ici l'approche proposée en termes de «corpus réflexifs » par Mayaffre (2002) : notre corpus est, à certains égards, conçu comme 
- Le Npr lui-même connaît, à travers des périodes-clé, une évolution de sens. Ce changement de sens peut être considéré, grosso modo, comme concomitant à l'accession du Npr Outreau à la notoriété.

- Le sens actuel communément partagé de Outreau peut être glosé par /erreur judiciaire/. Ce sens comporte un caractère dialogique, réfutatif, en ce qu'il repose à la fois sur des croyances collectives (existence d'un " réseau de pédophiles » à Outreau) et sur la réfutation collective ultérieure de ces croyances. On trouve également trace de ce dialogisme dans les textes.

De fait, il y a une historicité de l'affaire Outreau, et du sens de Outreau, et notre analyse en corpus prend cette historicité au pied de la lettre. Pour ce faire, nous avons partitionné le corpus en plusieurs sous-corpus correspondant à autant de périodes-clé, déterminées en partie sur la base de connaissances externes ${ }^{7}$. Nous y revenons plus bas.

Notre corpus est composé de textes (d'articles) de presse francophone, provenant de la banque de données d'archives de presse europresse.com et de ressources internet ${ }^{8}$. La sélection du corpus s'est effectuée sur la base de la seule présence du mot Outreau dans un texte donné, quelle que soit sa taille. Nous avons cependant éliminé, par un bref regard sur les textes, ceux dans lesquels Outreau ne relevait manifestement pas d'un des sens souhaités - notamment lorsque le nom renvoyait à une équipe sportive, et le texte à une autre thématique que la nôtre.

Ce premier choix interprétatif appelle un commentaire : nous avons cherché, par la récolte des textes, à rassembler la totalité des textes de presse rendus disponibles par europresse sur le thème choisi, mais non pas la totalité des occurrences de

un «tout-textuel sémantiquement auto-suffisant », même si, nous le verrons, des connaissances encyclopédiques interviennent nécessairement dans l'interprétation.

7. Cf. en annexe les «sources consultées». Ces connaissances externes, « hors corpus" donc, sont pour nous autant de ressources interprétatives.

8. Cf. en annexe le détail du corpus. 
Outreau, car c'est bien l'histoire de l'événement «affaire Outreau » que nous visions.

En revanche, un certain souci d'exhaustivité nous a conduite à conserver, dans les premières tranches du corpus du moins, tous types de textes publiés par la presse, y compris donc les courriers des lecteurs et les textes non journalistiques (analyse et tribunes d'experts ou d'hommes politiques), y compris aussi lorsque la référence à Outreau (l'événement qui nous occupe) se trouvait noyée dans des textes dont la thématique principale était toute autre, ce qui n'est pas sans influence sur la quantification des phénomènes, que nous évoquerons plus bas. Ce souci d'exhaustivité était guidé par l'objectif de cerner au plus près l'évolution du sens, nous l'avons dit, mais aussi la prégnance de la référence à Outreau comme symbole - car c'est bien de cela qu'il s'agit en dernière instance. Ainsi par exemple certain courrier des lecteurs se réfère, en passant, à Outreau comme comparant, mais à propos d'autres événements. Certain chroniqueur l'invoque pour appuyer une argumentation relevant d'un autre domaine.

En bref, notre corpus n'est donc pas à proprement parler du « discours journalistique »; il mêle plusieurs genres de la presse, mais aussi plusieurs types d'énonciateurs. Il est cependant supposé rapporter des faits, des récits, mais aussi des représentations, accessibles à la communauté francophone. Pour préciser encore ce point, ajoutons que nous avons conservé (après hésitation) les mots-clé qu'europresse attribue à chaque article pour archivage, mots-clé qui, certes, influent sur les résultats quantitatifs également, mais qui fournissent un précieux indicateur de la classification de l'événement, et parfois directement du sens de Outreau - par exemple le motclé erreur judiciaire en décembre 2005.

\subsection{Partition du corpus}

Nous avons partitionné notre corpus autour de périodes-clé, établies essentiellement sur la base de connaissances externes : investigations préalables, consultation de sites commentateurs de la presse et de l'ouvrage de Aubenas (2005). Les périodes retenues, marquées par des événements ou « rebondissements » de l'affaire sont les suivantes : 


\begin{tabular}{|l|l|l|l|}
\hline & Dates & Événement & $\begin{array}{l}\text { Taille } \\
\text { (mots) }\end{array}$ \\
\hline 1 & $\begin{array}{l}11 / 2001-\text { tout } \\
2002\end{array}$ & $\begin{array}{l}\text { « Découverte » d'un } \\
\text { réseau, arrestation de } \\
\text { « notables » }\end{array}$ & 43787 \\
\hline 2 & mai-juin 2004 & Procès de Saint-Omer & 1745743 \\
\hline 3 & $1-2 / 07 / 2004$ & $\begin{array}{l}\text { Attente du verdict du } \\
\text { procès de Saint-Omer }\end{array}$ & 11537 \\
\hline 4 & 3 à 8/07/2004 & $\begin{array}{l}\text { Verdict du procès de Saint- } \\
\text { Omer }\end{array}$ & 54153 \\
\hline 5 & $\begin{array}{l}2 / 12 / 2005 \text { à } \\
\text { mars-avril 2006 }\end{array}$ & $\begin{array}{l}\text { Procès en appel à Paris ; } \\
\text { suite et conséquences } \\
\text { (Commission d'enquête } \\
\text { parlementaire, ouverte le } \\
7 / 12 / 05 \text { ) }\end{array}$ & 26124 \\
\hline & Total (mots) & & 1881344 \\
\hline
\end{tabular}

On le voit, la taille des sous-corpus est disparate. On le voit aussi, des textes relevant de dates très proches (début juillet 2004) ont été répartis dans deux sous-corpus différents (3 et 4), ici sur la base de la consultation des textes eux-mêmes qui relèvent tendanciellement de genres et thèmes différents : le premier ( $1^{\text {er }}$ et 2 juillet) est centré sur l'événement " procès », le second (du 3 au 8 juillet) est composé de textes d'analyses et de commentaires de l'issue du procès.

Aux époques représentées peuvent être rattachés des sens différents de Outreau, plus ou moins stables et univoques : le Npr renvoie au début (2001-2002) majoritairement à un sens locatif: /ville/, assorti de la "facette»/(ensemble des) habitants/ ; il peut être glosé, dans ce sous-corpus également, et

9. Ce dernier sous-corpus est « ouvert» : nous avons également consulté et exploité notamment des articles «papier» pour confirmation des analyses - essentiellement pour observer si le sens ultime de Outreau restait constant (c'est bien le cas, cf. l'exemple [2]). Les interprétations quantitatives ne portent que sur la partie chiffrée correspondant aux textes en format électronique. 
Polysignifiance du toponyme

par la suite, par /affaire de réseau pédophile/ (sous-corpus 2), /procès de ce réseau/ (sous-corpus 3 et 4), avant de se stabiliser (sous-corpus 5) en /fiasco judiciaire/. Cette répartition maintient des zones de chevauchement : ainsi par exemple le sens locatif de Outreau ne disparaît jamais totalement.

\section{Contexte et éléments d'analyse}

\subsection{Contexte}

Comme nous l'avons exposé dans un article précédent ${ }^{10}$, et comme il apparaîtra dans notre développement, le point de vue général qui sous-tend notre appréhension du contexte est celui d'une interaction dynamique des différents paliers que sont le syntagme, la phrase, le texte (article de presse) et l'intertexte (chacun des cinq sous-corpus ; le corpus dans son ensemble).

Le corpus retenu nous fournit le contexte général d'interprétation du sens des occurrences de Outreau, celui qui voit évoluer ce sens, mais aussi celui qui le fait évoluer. Au sein du corpus, chacun des sous-corpus peut donner lieu à une comparaison des interprétations ; il sert également, de manière cumulative, d'éclairage chronologique aux autres.

L'analyse est outillée, nous l'avons dit, mais elle s'appuie également sur notre interprétation. En effet, le corpus demeure de taille suffisamment «humaine " pour que nous puissions le connaître: nous avons lu la quasi-totalité des textes ; l'analyse est donc sous-tendue par cette connaissance, qui intervient notablement dans l'interprétation - nous l'avons mentionné déjà lorsque nous avons évoqué la partition du corpus. Ainsi par exemple, en dehors des quelques cas où la distribution étroite d'une occurrence de Outreau nous guide de manière univoque vers sa signification (après Outreau), la plupart des occurrences de Outreau sont interprétées sur la base d'un contexte plus large, qui nécessite un va-et-vient entre contexte étroit sélectionné à l'aide du concordancier - SN du type [N1 de Outreau] par exemple - et contexte large - le texte (daté) lui-même parfois -, sans conduire toujours pour autant à une interprétation univoque.

10. Lecolle (2006). 
Ce type de passage d'un contexte étroit à un contexte large est illustré dans la suite de l'article (cf. en particulier $\S 4.1)$.

\subsection{Utilisation du concordancier}

Le logiciel AntConc, mis en place par Laurence Anthony, est téléchargeable librement ${ }^{11}$. Parmi les différentes fonctionnalités qu'il propose, nous n'en avons retenu que quelques-unes, à la fois facilement accessibles au néophyte et pertinentes pour notre projet. Nous avons essentiellement utilisé les listes de mots, leur fréquence et surtout leur rang de fréquence ${ }^{12}$, et, pour une analyse distributionnelle, l'accès par "mot-cible " (search term) et liste de mots-cible ${ }^{13}$, assorti d'un tri du contexte droit et gauche. En outre, comme nous le mentionnions ci-dessus, nous avons largement fait usage du lien hypertexte qui renvoie, à partir d'une occurrence, au contexte large (phrase ou paragraphe ; texte souvent, accessible grâce à «l'ascenseur »). Ces différents accès se faisant, au choix, sur un fichier (correspondant à un sous-corpus) ou sur plusieurs, nous avons ainsi pu comparer les sous-corpus, et, pour certains phénomènes, d'un point de vue quantitatif. La plupart des résultats chiffrés sont cependant traités ailleurs (sous excel): c'est le cas pour ceux qui ne peuvent être obtenus automatiquement à partir de seules chaînes de caractères, et qui résultent d'une interprétation humaine (cf. infra § 5).

\subsection{Angles d'attaque de l'analyse}

A titre exploratoire, nous avons retenu sept angles d'analyse ${ }^{14}$ dans le double but: (a) de déterminer l'interprétation sémantico-référentielle de Outreau, mais aussi et surtout de cerner ce qui fait évoluer ce $\mathrm{Npr}$, et notamment le caractère dialogique que nous relevions plus haut; (b) de mettre en

11. Cf. la rubrique « logiciel et corpus » infra.

12. Une fois éliminés les mots grammaticaux, ainsi que certains adverbes et auxiliaires.

13. Par exemple les mots-cible Outreau, réseau, le groupe de «mots-cible » le réseau, un réseau, existence d'un réseau (cf. infra § 3.3 et $\S 4.1$ ), ou encore la liste de mots-cible présumé, supposé (réseau) (cf. infra § 3.3).

14. Nous ne développons ici que les cinq premiers. 
Polysignifiance du toponyme

évidence son installation comme une évidence et comme un type ("fiasco judiciaire »), dans la compétence discursive collective. Notons que les points (a) et (b) sont susceptibles d'interagir.

Chacun des points envisagés rassemble des phénomènes linguistiques qui résultent d'ajustements interprétatifs, guidés par une familiarité avec le corpus, notamment la constatation de la récurrence de faits linguistiques interprétables comme des indices ou des marqueurs - ainsi, les prépositions avant, après, malgré marquent une interprétation événementielle du toponyme (Godard \& Jayez 1996). Ils procèdent également de notre conception du Npr, comme nom d'un individu susceptible de voir évoluer son sens et changer sa signification. Enfin, ils s'articulent avec le projet global d'envisager le Npr dans un cadre discursif historicisé.

1. Le premier angle d'analyse renvoie à ce qui, dans la signification catégorielle du Npr, est susceptible d'une contrepartie syntaxique et distributionnelle, utilisée ici comme indice minimal: Outreau comme lieu, comme événement (procès ou "affaire » - terme lui-même sous-spécifié) connaissent parfois une telle contrepartie, cf. [3]. C'est ici que nous procédons à l'analyse de la distribution et/ou fonction de Outreau: Outreau en Sprép, et dans la structure [N1 de Outreau] ; Outreau en fonction sujet ou COD, par exemple. Cet indice, dans certains cas accessible directement au moyen du concordancier, comme la suite après Outreau, nécessite luimême cependant le plus souvent une interprétation en cadre phrastique: on connaît le caractère sous-spécifié des prépositions à et de, différentes en ceci de malgré ou après ; la signification de Outreau en fonction sujet dépend naturellement du sens du verbe (de fait, elle correspond le plus souvent au sens /(ensemble des) habitants/, cf. [4]). Nous revenons plus loin (§ 4.2) sur la portée de cet angle d'attaque de l'analyse.

[3] [Les magistrats] On leur demande, après Outreau, d'être plus attentifs aux placements en détention, mais on leur reproche de ne pas céder à la pression de la police dès qu'ils n'incarcèrent pas, comme l'ont montré plusieurs affaires récentes. (Le Monde, entretien, 5/07/04) 
[4] Outreau vit des jours tragiques (en titre, La Croix, 14/01/02)

2. Un deuxième angle d'analyse, mené au niveau de la phrase et/ou du texte, renvoie à ce que nous avons nommé « sens » du Npr. Les comparaisons (Outreau comme comparé) et les énumérations entrent dans la construction de ce sens : ainsi Outreau est souvent rapproché de Dutroux [5] et [6] ${ }^{15}$, mais aussi parfois (et plus tard) d'erreurs judiciaires et/ou médiatiques comme celle de "Bruay en Artois ", «du RER D », «l'affaire Alegre» [7], ou celle «du bagagiste de Roissy ». Cette analyse marque l'évolution du cadre de référence de Outreau, et participe au repérage de Outreau comme /erreur judiciaire/ dans la dernière étape.

[5] [affaire de pédophilie révélée en novembre 2001 à Outreau]. A ce jour, près d'une vingtaine de personnes ont été mises en examen dans cette affaire. L'existence d'un réseau francobelge - on repense inévitablement à l'affaire Dutroux (photo) - est avérée, avec vente de photos et de films vidéo. (Le Monde, 30/03/02).

[6] Outreau et Dutroux, l'ombre portée de la pédophilie. (Le Monde, 21 mai 2004)

[7] «Le vacarme de la presse devient insupportable dans les prétoires. Markovic, Alègre, Carpentras, aujourd'hui Outreau, cela suffit ! » (Le Monde, 7/07/04, un avocat)

3. Le sens de Outreau est également appréhendé par le biais de ce que nous avons nommé ailleurs ${ }^{16}$ le «scénario" de l'événement. Il s'agit principalement ici des «actants» de Outreau comme événement: les enfants, la principale accusatrice (nommée par son nom), la boulangère, l'huissier (présentés comme des personnages, par le biais de noms de rôles), les (dits) "notables » etc. Nous avons donc observé systématiquement, à travers la manière dont les mots apparaissaient autour de Outreau dans les textes, l'émergence et l'installation de ces personnes comme « actants » d'un scénario

15. On observe ici un fait important: avec cette catégorie de structures (comparaison et énumération), la différence catégorielle des $\mathrm{Npr}$ (lieu / personne) s'annule.

16. Lecolle (à paraître). À propos des événements Tchernobyl, Tiananmen, (le festival de) Cannes, (les jeux olympiques) d'Atlanta. 
attaché à un Outreau événementiel - on comparera à cet égard [8] à [9]. Ce scénario n'est bien sûr pas le même selon les époques : dans le récit, notables disparaît, enfants reste présent tandis que juge et magistrats par exemple interviennent par la suite : on le constate par le recours aux décomptes de fréquence avec Antconc ${ }^{17}$.

[8] Le 12 avril, Mme Godart, boulangère à Outreau, est placée en détention. (Le Figaro, 14/01/02)

[9] [Affaire d'Outreau Le juge et la " menteuse »] Et cette maladie qu'est la détention provisoire ajoute à cette affaire un relief tout particulier. Était-il indispensable de garder la boulangère et l'huissier en prison? (Le Point, 27/05/04, interview de Maître Leclerc, avocat)

4. Un événement est souvent associé à des conséquences et des causes. Ainsi, Outreau, en tant qu'événement social, entre dans ce que Quéré (1994) nomme des «textures causales». Cette présentation de la causalité au sens large (cause, but, conséquence, voire symptôme ${ }^{18}$ ), tout en signalant, à un premier niveau, le sens événementiel du mot Outreau, "cadre » cet événement, selon les termes utilisés, en le ramenant à un ou des domaines de référence: "pédophilie ", "problème médiatique », " problème judiciaire », voire "fait de société ». C'est en ce sens qu'il participe à la construction du sens événementiel de Outreau.

[10] «L'inceste passé à l'acte est au cœur des systèmes maltraitants, très répandus, et dont cette affaire d'Outreau apparaît comme un révélateur. » (Interview d'un psychiatre et psychanalyste, Le Monde, 12/06/2004).

17. Notons le singulier de juge et le pluriel de magistrats. De telles différences ont une certaine importance (indirecte cependant dans le cadre de ce que nous présentons ici), ce pourquoi nous nous sommes généralement gardée de lemmatiser les mots dans la recherche automatique.

18. Cf. Nazarenko (2000), qui utilise le terme générique de «causalité » pour renvoyer aux relations de cause et conséquence. En effet, l'auteur remarque que ces relations logiques inverses sont souvent discursivement mêlées. Nous faisons la même observation avec «but » et "symptôme" (cf. le terme de révélateur en [10]). Faute de place, nous ne développons pas davantage ce point. 
[11] Pour éviter un nouvel Outreau (en titre). [...] Le cauchemar d'Outreau s'achève. [...] La première cause fondamentale de ces errements est la procédure judiciaire elle-même. (Les Échos, 2/12/05)

5. Le cinquième angle d'analyse renvoie aux faits ressortissant du dialogisme : on l'a dit, en dernière instance (2005-2006), et même dès le procès de Saint-Omer (sous-corpus 2, 3 et 4), le phénomène Outreau est considéré comme une erreur judiciaire ; plus encore, le Npr Outreau lui-même renvoie à /l'erreur judiciaire par excellence/. Ce terme d'erreur judiciaire contient dans son sens un caractère dialogique, réfutatif, qui peut être considéré ici comme le résumé de l'histoire d'Outreau ellemême. C'est dans cette optique que sont recherchés ici de manière automatique des marqueurs supposés relever de l'expression du doute, de la modalisation (ou au contraire leur absence), portant non plus sur Outreau même mais sur les faits qui lui sont attachés : les guillemets (sur Outreau, mais aussi sur des termes-clé comme notable et surtout réseau ${ }^{19}$ ); les adjectifs comme supposé(s), présumé(s) (réseau, notables, réseau de notables) etc. ; les noms thèse, hypothèse, histoire, piste (du réseau) et, à l'opposé, existence (d'un réseau) (cf. [12], comparé à [13]); les déterminants (le réseau pédophile d'Outreau vs un réseau pédophile) entrent dans cette analyse.

[12] D'ores et déjà, on constate que l'affaire d'Outreau n'est pas une «banale » histoire de ballets roses, comme on disait autrefois [...]. Ici, un véritable réseau a été démantelé. Des notables y côtoyaient des prolétaires. (Le Figaro, 14/01/2002).

[13] Les deux affaires [Dutroux et Outreau] réunissaient toutefois tous les ingrédients dont sont immanquablement friands les médias: un contexte sulfureux, un tabou soulevé, la pédophilie, une histoire de " réseau ", des personnages hauts en couleur (la «boulangère ", le " taxi », etc.). (Le Figaro, 13/03/06).

Les deux derniers angles d'attaque s'intéressent à l'acquisition de la notoriété du Npr, qui nous semble être concomitante à

19. Réseau de notables par exemple n’est plus employé que modalisé en maijuin 2004. 
Polysignifiance du toponyme

l'installation du sens ultime de Outreau comme /erreur judiciaire/. Nous ne développons pas ici ces points, dont l'interprétation prend notamment en compte des indices typodispositionnels comme la position du $\mathrm{Npr}$ en titre de journal et sa signification dans cette position, l'utilisation de erreur judiciaire comme mot-clé dans l'archivage de europresse, ou encore la présence d'antonomases (cf. ci-dessus [2] et [11]). Cette figure sanctionne l'installation d'un type attaché au référent du Npr, ici Outreau comme parangon d' «erreur judiciaire ». Elle n'est présente que dans les textes de la dernière période.

Avec ces angles d'analyse nous proposons autant de points de vue, différents mais complémentaires, destinés à résoudre le problème interprétatif posé - polysignifiance, évolution du sens du $\mathrm{Npr}$-, par le biais d'un environnement étroit ou élargi. Ces différents points, leur pertinence comme leur articulation peuvent naturellement être discutés. Mais nous centrerons notre discussion sur certaines difficultés théoriques et pratiques de leur mise en œuvre ( $\S 4)$ et sur l'apport et les limites d'une approche quantitative ( $§ 5$ ), en espérant cependant poser en filigrane des jalons de cette articulation.

\section{Discussion}

Cette section revient sur certains points d'entrée de l'analyse. A travers les points abordés, c'est, de diverses manières, la question de l'empan pertinent pour l'interprétation, la recherche de «marqueurs » et d'indices interprétatifs, et le rôle du contexte qui seront discutés.

Le concordancier, employé a minima comme simple outil de recherche dans le texte en version électronique (repérage d'occurrences du mot réseau et de son déterminant dans un SN (§ 4.1), distribution de Outreau en Sprép (§ 4.2) ou recherche de Npr connus préalablement par exemple (§ 4.3)), ou dans des fonctionnalités plus spécifiques (concordances), est un précieux outil de repérage de certains marqueurs. Mais il ne pallie pas le problème de l'équivocité ou de la sousdétermination que nous avons à rencontrer (§ 4.2). Et il n'est de toutes façons que de peu d'utilité dans certains cas, comme 
celui de la causalité : sans développer plus avant, signalons simplement que celle-ci, omniprésente dans les textes, y prend dans les faits des tours très divers : les exemples [1] et [3], notamment, donnent lieu à une interprétation en termes de causalité. En conséquence, celle-ci ne relève pas d'un simple "repérage ", et suppose une interprétation appuyée sur un empan large (palier du paragraphe au moins, ou encore rapport entre titre et texte d'un article).

\section{1. Présupposition d'existence (le réseau pédophile), et paliers d'interprétation}

Revenons tout d'abord sur un exemple simple d'analyse, pour illustrer le va-et-vient, mentionné à plusieurs reprises, entre contexte étroit (ici, le syntagme comme premier palier d'interprétation) et contexte plus large. Parmi d'autres, l'angle d'analyse 5 du $\S 3.3$ relève de cette approche.

Connaissant, par l'exploration du corpus, l'importance du mot réseau dans la construction de "l'affaire d'Outreau » ${ }^{20}$, et en particulier le rôle de la présupposition existentielle attachée à ce nom, notre premier mouvement a été de décompter et comparer les syntagmes définis et indéfinis contenant réseau (le vs un réseau ${ }^{21}$ ). Mais cette analyse s'avérait trop sommaire: d'une part parce que, au palier syntagmatique supérieur, le SN défini récurrent l'existence d'un réseau comporte bien le SN enchâssé un réseau; d'autre part parce que le SN l'existence d'un réseau lui-même se trouve, selon les contextes, textuellement affirmé, mis en doute ou nié, comme le montrent les exemples suivants (cf. également [5]) :

[14] L'affaire d'inceste familial a révélé l'existence d'un réseau. (L'Humanité, 14/01/02)

[15] Quelle que soit son issue, le procès d'Outreau aura réveillé de vieux fantasmes sur le Nord. Le traitement par les médias aurait-il été le même si les faits s'étaient déroulés ailleurs ? Bien sûr, dans la région, le chômage et la délinquance sont au plus haut, les résultats au bac et l'espérance de vie au plus

20. Quatrième rang de fréquence dans le sous-corpus 1.

21. Dans le sous-corpus 1, un réseau: 25 occurrences, le réseau: 47, ce réseau : 7. 
Polysignifiance du toponyme

bas. De là à juger «naturelle » l'existence d'un réseau pédophile, il y a un pas... (L'Express, 31/05/04)

On le voit, si, aux premiers paliers, les «marqueurs » réseau, un réseau, l'existence d'un réseau guident notablement l'interprétation, celle-ci relève en définitive d'un empan plus large, et variable : phrastique en [14] avec le verbe révéler au passé composé ; de l'ordre du paragraphe en [15], où apparaît un ton globalement distancié, dont les différentes formes (ponctuation, lexique notamment) ne sont d'ailleurs pas analysables par simple sommation.

\section{2. Univocité, équivocité et sous-détermination des marqueurs : les prépositions}

L'interprétation rencontre une autre difficulté, celle de l'équivocité, liée à la sous-détermination de certaines prépositions.

L'angle d'attaque 1 , qui s'attache à la distribution de Outreau au niveau du syntagme, et particulièrement en Sprép, nous paraît s'imposer, pour cerner la « signification minimale » (la catégorisation) de Outreau. Pourtant, si certaines prépositions agissent de manière discrète et discriminante, jouant alors un rôle de " marqueur », d'autres conduisent à une interprétation équivoque ou continue.

Ainsi, avec des prépositions telles que malgré ou des prépositions temporelles (jusqu'à, avant, après), le Sprép permet de révéler les significations événementielles de Outreau dans leur ensemble (sans pour autant conduire réellement au " sens » lui-même), qui peuvent alors être comptabilisées.

De son côté, la préposition à, associée à un Npr de lieu, renvoie souvent à une valeur locative (cf. [8] supra), mais c'est avec ce " souvent» (et son corollaire - « pas toujours ») que les ennuis commencent. Que dire en effet du à Outreau de [1], déjà mentionné ? Et de ceux de [16] et [17] - lors du procès à SaintOmer, en mai-juillet 2004 ?

[16] Des experts défilent à Outreau (Libération, 5/06/04)

[17] À Outreau, la parole est aux victimes (La Croix, 1/06/04)

Incontestablement, à Outreau doit, dans ces deux derniers exemples, être interprété comme temporel (/lors du procès 
d'Outreau/) et Outreau lui-même comme événementiel, nous semble-t-il. Une sélection mutuelle - une co-construction - agit ici, en précisant le sens de la préposition conjointement à celui du Npr, l'interprétation du syntagme lui-même relevant en réalité du palier d'analyse qu'est le texte (daté) dans le cadre du sous-corpus.

Si la préposition à révèle ici son équivocité, plus problématique encore est le cas de de, déjà évoqué pour sa sous-détermination. Dans le cadre du SN [N1 de Outreau], la préposition peut prendre plusieurs valeurs. Elle introduit notamment :

- une catégorisation : la ville d'Outreau - Outreau est une ville ;

- une qualification : le fiasco d'Outreau - Outreau est (vu comme) un fiasco ;

- une "appartenance »: les enfants d'Outreau - les enfants appartiennent à l'ensemble des habitants d'Outreau ;

- un rapport actanciel, où Outreau (mais dans quel sens ?) fait figure d'agent : les leçons d'Outreau ;

- un rapport actanciel, qui ici renvoie à Outreau événementiel : les accusés d'Outreau.

C'est ainsi que [N1 de Outreau] donne lieu à des équivoques : le prêtre-ouvrier d'Outreau ou les enfants d'Outreau sont-ils des habitants du lieu Outreau, des actants (respectivement accusé et victimes) du réseau pédophile (d'Outreau), des actants du procès (d'Outreau) à Saint-Omer ou, à un niveau générique, des actants de «l'affaire» ? Ou encore tout à la fois, dans une interprétation non-discriminante et continue ? En résumé, à l'équivocité et la sous-détermination de la préposition de s'ajoutent ici l'équivocité et la sous-détermination de Outreau.

\subsection{Rôle des noms propres dans l'interprétation}

Selon l'« angle d'attaque » 2, nous examinons le rapprochement de Outreau avec d'autres Npr, rapprochement exprimé par le biais de structures syntagmatiques telles que l'énumération et la comparaison $^{22}$. Il s'agit ici de rendre compte de son intégration

22. Ces deux catégories de structures, ailleurs distinctes, pourraient ici donner lieu, le cas échéant, à une annotation unique, motivée par leur proximité sémantique dans l'usage spécifique que nous en faisons. 
Polysignifiance du toponyme

dans des réseaux de Npr, comme comparé. Nous avons vu que de tels réseaux transcendent la différence de catégorie entre toponymes et anthroponymes, en réalité tous marqués socialement, à un autre niveau, comme des "affaires". Indépendamment même du repérage des structures, sans doute systématisable dans une analyse autrement outillée que la nôtre, l'interprétation repose sur la connaissance de ces $\mathrm{Npr}$ - des événements ou des personnes qui s’y rattachent. La difficulté théorique de cet élément interprétatif, et sa difficulté pratique pour une approche automatisée ne doivent pas masquer son importance dans l'interprétation, au-delà du seul cas que nous étudions ici: les textes (notamment journalistiques) sont peuplés de $\mathrm{Npr}$, dont le rôle est loin d'être simplement dénominatif. Comme nous cherchons à le montrer pour Outreau lui-même, ces Npr apparaissent potentiellement chargés de sens; ils ont une fonction résomptive, emmagasinant les connaissances, et les discours énoncés à leur propos, et sont, par voie de conséquence, porteurs d'implicite.

Toutefois, nous avons pu montrer ailleurs ${ }^{23}$ qu'une connaissance préalable des Npr eux-mêmes et de leur référent n'est pas toujours nécessaire à l'interprétation occurrente. Lorsque ceux-ci apparaissent groupés en contexte phrastique (comme par exemple en [6] et [7]), chacun d'entre eux agit sur l'interprétation des autres, par un éclairage mutuel, appuyé par une structure syntagmatique - ici comparaisons et énumérations - qui manifeste leur rapprochement paradigmatique.

Il n'en demeure pas moins que le repérage des Npr, que celui de la structure même où ils apparaissent, ne sont que le premier pas de l'interprétation : Outreau comparé à Dutroux ne construit pas le même sens selon le contexte, on le voit en comparant les exemples [5] et [13]. En effet, bien que la référence à Dutroux reste présente dans l'ensemble du corpus (cf. §5 infra), cette référence comparative est systématiquement, à partir de 2004, "de second niveau » - oppositive, distanciée et modalisée -, dès lors que la comparaison «de premier niveau », présentant deux affaires de «réseau pédophile », n'est plus valable. Un empan large - contexte

23. Lecolle (2006 et à paraître). 
phrastique, voire textuel - est ici nécessaire pour l'utilisation adéquate de la comparaison Outreau-Dutroux comme élément d'interprétation.

\section{Quantifier, oui mais quoi ?}

Il découle de ce qui précède ( $§ 3.3$ et 4 ) que nos critères d'analyse ne doivent et ne peuvent être tous quantifiés. L'expérience de ce travail nous a cependant montré que des analyses quantitatives sont, dans certains cas, d'un appui considérable. D’une manière générale, et quand c'était possible, nous avons donc eu recours à des approches quantitatives et " qualitatives » de manière interactive et cumulative, l'analyse supposant, ici encore, un va-et-vient entre sélection de marqueurs, résultats et interprétation nouvelle.

Illustrons ceci, à partir de trois exemples.

Tout d'abord, la quantification nous a permis de comparer les différents sous-corpus du point de vue de deux des principales «étiquettes » de significations que nous avons attribuées au Npr Outreau entre la première et la dernière période. Les résultats que nous rapportons sont ceux qui reposent essentiellement sur le point 1 - la syntaxe et la distribution de Outreau. Dans les périodes 1 et 5 , ces significations sont, respectivement ${ }^{24}$ :

- /lieu/ : 53,88\% (sous-corpus 1) et 7,43\% (sous-corpus 5) ;

- /erreur judiciaire/ : 0\% (sous-corpus 1) et 38,86\% (souscorpus 5).

De fait, l'évolution de ces chiffres est significative : elle sanctionne effectivement l'évolution de la signification du Npr entre les deux pôles ultimes, et univoques, /lieu/ et /erreur judiciaire/. Il reste pourtant un pourcentage important, en particulier pour le sous-corpus 5 (53,71\%), que nous ne pouvons caractériser de manière précise pour des raisons que, faute de place, nous ne faisons ici qu'évoquer : pour une part du moins, il s'agit globalement de zones d'incertitude et de chevauchement des sens que l'analyse distributionnelle ne peut

24. Dans chacun des sous-corpus, le total des occurrences de Outreau dans telle signification rapporté en pourcentages au total des occurrences de Outreau, soit ici respectivement 206 et 175 occurrences. 
Polysignifiance du toponyme

élucider (cf. supra, notamment notre commentaire de l'exemple [1]). Une autre part renvoie, en toute vraisemblance, aux sens événementiels ou apparentés tels que /le procès d'Outreau/, /l'affaire d'Outreau/ qui ne peuvent pas non plus être atteints simplement par les outils d'analyse de l'angle d'attaque 1 .

En tout état de cause, il convient de signaler que les résultats donnés ne sont pas obtenus directement avec le concordancier : à partir d'occurrences de formes de Outreau dans son contexte étroit (fenêtre de deux mots à gauche du motcible Outreau), nous intervenons en fait ici pour établir, par abstraction, des catégories à partir de ces formes - ce qui suppose de séparer des formes proches et de rapprocher des formes différentes : sont par exemple rapprochés HLM, maire, habitants d'Outreau, mais aussi habiter à Outreau d'un côté, fiasco, naufrage, scandale, horreur (judiciaire) ${ }^{25} d^{\prime}$ 'Outreau, de l'autre. C'est alors l'ensemble de ces suites que nous ramenons au nombre d'occurrences de Outreau dans un sous-corpus donné.

Le deuxième exemple, déjà abordé sous «l'angle d'attaque » 3 du $\S 3.3$ (scénario et actants), se rapporte aux rangs de fréquences des mots des sous-corpus comparés. Ceuxci peuvent être, en effet, hautement révélateurs dans les premiers rangs, et venir en appui d'autres éléments d'analyse : ainsi Outreau acquiert en 2005-2006 la première place (contre la $8^{\text {ème }}$ dans le sous-corpus 3 par exemple), suivi de affaire, ce qui est tout à fait significatif eu égard à l'installation de la notoriété de Outreau comme fait public.

Enfin - dernier exemple -, nous avons eu l'occasion de signaler, dans le cadre de l'angle d'analyse 2 (se rapportant aux réseaux de $\mathrm{Npr}$ ), que la référence à Dutroux comme comparant était révélatrice du cadre de référence de Outreau comme fait public. Celle-ci apparaît 28 fois en 2001-2002, 123 fois en maijuin 2004 et 6 fois en 2005-2006, soit, en fréquences relatives ${ }^{26}$, respectivement $0,064 \%, 0,007 \%$ et $0,023 \%$. On le voit, la

25. Et, plus généralement, les termes synonymes.

26. Occurrences de Dutroux rapportées en pourcentages au nombre de mots du sous-corpus. Les pourcentages sont faibles, certes, mais intéressants en termes comparatifs. 
référence à Dutroux reste présente tout au long de l'histoire de Outreau, bien que de manière plus importante dans le souscorpus 1 . Ici cependant, le seul critère absence vs présence, et son chiffrage même, bien que pertinents, apparaissent insuffisants pour l'analyse, puisque, nous l'avons dit (§ 4.3), ce Npr ne construit pas le même sens selon les périodes.

\section{Conclusion}

Notre travail se situe dans le cadre d'une approche dynamique du sens et de la signification, opposée à une conception univoque et fixe du sens du Npr.

L’analyse s'attachait au repérage de l'évolution du sens d'un toponyme, Outreau, de nom de ville à nom d'événement public - un "fiasco judiciaire » - faisant figure, pour l'ensemble de la société, de référence négative. Avec l'objectif de décrire en diachronie le sens de Outreau, nous avons constitué un corpus de textes de presse, envisagé dans son historicité. La partition de ce corpus en plusieurs sous-corpus, correspondant à autant d'étapes de l'histoire du Npr et de son cadre de référence, reposait sur une connaissance préalable de l'événement relaté.

Nous avons proposé, à titre exploratoire, un croisement de plusieurs critères d'analyse, comme éléments de repérage du/des sens et des significations, mais aussi comme repérage des faits discursifs qui font évoluer ce sens. Cette approche multi-critères, nécessitant un va-et-vient entre contexte étroit (syntagme) et large (texte, sous-corpus), n'apparaît possible que dans le cadre d'un corpus de taille limitée où l'interprétation, secondée et guidée notablement par un concordancier, intervient néanmoins, et de différentes manières, à tous les niveaux. Elle nous a permis de dégager quelques éléments solides manifestant le changement de sens et de signification de Outreau - dans son environnement distributionnel, et, à un niveau plus global, dans son cadre de référence.

L'analyse s'attachait au cas du Npr, et d'un $\mathrm{Npr}$ particulier, mais elle peut être élargie à toute approche dialogique de l'enrichissement du sens qu'une entité (un individu) est susceptible d'acquérir dans les textes - ici de 
presse, ailleurs de fiction -, à travers les représentations qui en sont données directement (par la nomination ou la prédication) ou indirectement (par les comparaisons, les énumérations ou le jeu des actants).

\section{Références bibliographiques}

Cislaru G. (2005). Étude sémantique et discursive du nom de pays dans la presse française avec référence à l'anglais, au roumain et au russe, thèse de doctorat, Université Paris III-Sorbonne Nouvelle.

Fuchs C. (1991). «L'hétérogénéité interprétative », in H. Parret (dir.) Le sens et ses hétérogénéités. Paris : Éditions du CNRS, 107-120.

Gary-Prieur M.-N. (1994). Grammaire du nom propre. Paris : Presses Universitaires de France.

Godard D. \& Jayez J. (1996). "Types Nominaux et Anaphores: le cas des objets et des événements ", in W. De Mulder, L Tasmowski-De Ryck \& C. Vetters (éds), Anaphores temporelles et (in-)cohérence. Amsterdam/ Atlanta : Rodopi, 41-58.

Habert B. (2005). Instruments et ressources électroniques pour le français. Paris / Gap : Ophrys.

Lecolle M. (2004). «Toponymes en jeu : diversité et mixage des emplois métonymiques de toponymes", Studii si cercetari filologice 3: 5-13 (Université de Pitesti, Roumanie).

Accessible en ligne sur le site La métaphore en question à l'adresse : http://www.info-metaphore.com/articles/lecollejeu-toponymes-metonymie-emploi-metonymique.html

Lecolle M. (2006). «Polyvalence des toponymes et interprétation en contexte », in G. Achard-Bayle (éd.) Pratiques 129/130 : 107-122.

Lecolle M. (à paraître). «Éléments pour la caractérisation des toponymes en emploi événementiel », Actes du Colloque international Représentations du sens linguistique III, Bruxelles, du 3 au 5 novembre 2005. 
Leroy S. (2004). Le nom propre en français. Paris / Gap : Ophrys.

Mayaffre D. (2002). «Les corpus réflexifs: entre architextualité et hypertextualité », Corpus 1, [en ligne] http://revel.unice.fr/corpus/document.html?id=11 (consulté le 29/10/04).

Nazarenko A. (2000). La cause et son expression en français. Paris / Gap : Ophrys.

Pincemin B., Issac F., Chanove M. \& Mathieu-Colas M. (2006). "Concordanciers : thème et variations ", in J.-M. Viprey (éd.), 8èmes JADT 06. Besançon : Presses universitaires de Franche-Comté, vol. 2, 773-784.

Quéré L. (1994). "L'événement "sous une description": contraintes sémantiques, croyances stéréotypiques et "natural facts of life as a morality" ", Protée. Théories et pratiques sémiotiques, Département des Arts et Lettres de l’Université de Québec, Chicoutimi, vol. 22, 2 : 14-28.

Rastier F. (1998). «Le problème épistémologique du contexte et le statut de l'interprétation dans les sciences du langage », Langages 129 : 97-111.

Rastier F. (2001). Arts et sciences du texte. Paris: Presses Universitaires de France. 
Polysignifiance du toponyme

\section{Annexes}

\section{Sources consultées sur " l'affaire d'Outreau "}

Aubenas F. (2005). La méprise: l'affaire d'Outreau. Paris, Seuil.

Cara T. "'Affaire d'Outreau' : Après le 'délire’ médiatique, l'amnésie collective ", publié le mercredi 14 décembre 2005 sur ACRIMED (Action, critique, médias), [en ligne] http://www.acrimed.org/

Filio N. «D'Outreau à Dutroux, la terrible méprise », publié le 24 mai 2004 sur le site de Arrêt sur Images, [en ligne] http://www.france5.fr/asi/006052/36/113843.cfm

Filio N. "A quand les excuses des médias ?", publié le 18/12/05 sur le site de Arrêt sur Images, [en ligne] http://www.france5.fr/asi/006052/36/113843.cfm

\section{Logiciel et Corpus}

Antconc, téléchargeable sur le site de Laurence Anthony [en ligne] http://www.antlab.sci.waseda.ac.jp/software.html

Archives Europresse.com : Presse francophone, hebdomadaires et quotidiens : l'Humanité, le Monde, le Figaro, l'Express, le Nouvel Observateur, le Point, La Croix, Libération, Les Échos, La Tribune, Le Soir (quotidien belge). [en ligne] http://www.europresse.com

Articles de presse provenant des archives internet de différents médias (TF1, RTL, Le Parisien).

En complément (2005-2006), articles papier (Politis, Télérama, Libération, Le Monde). 